\title{
The COMT Val158Met Polymorphism and Exploratory Behavior in Bipolar Mania
}

\author{
Arpi Minassian Jared W. Young Mark A. Geyer John R. Kelsoe William Perry \\ Department of Psychiatry, University of California San Diego, San Diego, CA, USA
}

\section{Keywords}

Catecholamines · Bipolar disorder - Arousal · Mania

\begin{abstract}
Background: The catechol-O-methyltransferase (COMT) Val158Met gene influences cognition and behavior in psychiatric illnesses; its low-activity allele, methionine (Met), may be associated with behavior reflecting catecholamine overactivity. Heightened motor activity and increased positive valence are central features of bipolar disorder (BD) and have been quantified in the human Behavioral Pattern Monitor (hBPM), an exploration paradigm based upon the rodent open field. We examined whether hBPM behavior was related to the COMT gene in a small sample of manic BD patients. Methods: Twenty-six acutely hospitalized manic BD patients were genotyped for the COMT Val158Met polymorphism and tested in the hBPM, an unfamiliar room containing novel objects. Movements around the hBPM and object interactions were video-recorded for $15 \mathrm{~min}$ and rated. $\boldsymbol{R} \boldsymbol{e}$ sults: Met homozygote BD patients demonstrated significantly more interactions with multiple objects and more time spent with objects in the hBPM. Valine (Val) homozygote patients exhibited the least object exploration, while heterozygote patients demonstrated intermediate levels.
\end{abstract}

Conclusion: This preliminary study suggests that arousal and positive valence are influenced in a linear fashion by COMT, presumably due to increased catecholamine in frontal regions, but these findings require replication in a larger sample. The hBPM can enable cross-species and transdiagnostic studies to inform neurobiology of psychiatric disorders.

(c) 2017 S. Karger AG, Basel

\section{Introduction}

In recent years, the National Institute of Mental Health (NIMH) outlined the Research Domain Criteria (RDoC) initiative, a new way of understanding psychopathology based on dimensions of functioning [1]. The RDoC program hopes to characterize patients dimensionally using domain-specific behaviors and identify biomarkers linked to neural function of said domains to develop more target-specific therapeutics. With respect to biomarkers, gene variations relevant to catecholamine signaling and its reuptake have been linked with psychiatric disorders. For example, the mania of bipolar disorder (BD) may be influenced by dysregulation of the dopamine (DA) system, and blocking DA reuptake (the DA

\section{KARGER}

(c) 2017 S. Karger AG, Basel

E-Mail karger@karger.com

www.karger.com/mnp
Arpi Minassian, PhD

Department of Psychiatry, University of California San Diego

200 West Arbor Drive, Mailcode 8620

San Diego, CA 92109-8620 (USA)

E-Mail aminassian@ucsd.edu 
transporter; DAT) models several aspects of mania in animals [2]. Accordingly, genes that regulate synaptic DA reuptake such as the DAT gene have been linked with $\mathrm{BD}$ [3]; however, the influence of other synaptic DA clearance-related genes on phenotypes of $\mathrm{BD}$ has been relatively understudied.

The catechol-O-methyltransferase (COMT) gene plays an important role in the metabolic inactivation of DA and norepinephrine in regions with a paucity of DAT expression, specifically in the frontal cortex [4]. A common functional polymorphism of this gene involves an amino acid substitution of methionine (Met) for valine (Val) which changes the activity of the enzyme. Val homozygotes have 3-4 times higher COMT enzyme activity, resulting in increased catabolism of catecholamines in frontal regions compared to Met homozygotes [5]. Given the importance of the frontal cortex in cognition and decision-making - behaviors impaired in BD [6] - understanding the role of frontal DA clearance enzyme COMT in $\mathrm{BD}$ patients may be critical for developing targeted treatments.

The preponderance of the COMT literature, primarily focused on schizophrenia, links impaired cognition with the Val allele of the Val158Met polymorphism. For example, Val/Val homozygotes have lower sensorimotor gating levels [7]. In contrast, the Met allele has been associated with aggressive behavior [8] and suicidality [9] in schizophrenia patients. These observations are consistent with the hypothesis proposed by Bilder et al. [10] that the Met allele confers an increase in tonic catecholamine function, which putatively leads to a decreased ability to plan adaptive responses to novel situations. Thus, features of behavioral overactivity may be associated with the presence of the Met allele.

Overactivity, defined as an increase in goal-oriented activities, is a characteristic feature of the manic phase of $\mathrm{BD}$ and is often coupled with a tendency toward impulsivity, risky behavior, and social norm violations (e.g., hypersexuality, spending, traveling, or stealing) [11]. To objectively quantify this important feature of mania, we designed the human Behavioral Pattern Monitor (hBPM), a translational paradigm based upon our work with the rodent Behavioral Pattern Monitor (BPM) [12]. The $\mathrm{hBPM}$ enables the characterization of overactive exploratory behavior in response to novel stimuli $[13,14]$. We reported that manic $\mathrm{BD}$ patients exhibit a unique behavioral profile in the hBPM when compared to patients with schizophrenia and non-patient comparison subjects, exhibiting increased (1) novel object interactions, (2) multiple object interactions (MOIs), and (3) forays into areas of the hBPM that contain novel objects $[13,14]$. The RDoC behavioral constructs [15] that most closely align with this profile - which is not entirely specific to mania and is also observed in other conditions with putative catecholamine dysregulation (ADHD, substance use disorders) - include the dimensions of arousal (specifically motor activity) and positive valence (specifically approach motivation). Although we have reported evidence that DAT may play a role in the manifestation of these phenotypes [16], to date the putative contribution of COMT activity has not been evaluated.

The current study examined the underlying biology of this profile in $\mathrm{BD}$ by testing the hypothesis that the Met allele of the COMT Val158Met gene would be associated with increased exploratory behavior in BD mania patients. This finding would underscore the relationship between catecholaminergic function and the RDoC domains of arousal and positive valence.

\section{Methods}

\section{Subjects}

This study was approved by the UCSD Human Research Protections Program. Twenty-six (16 male, 10 female, 22 Caucasian) acutely hospitalized inpatients with SCID (Structured Clinical Interview for DSM-IV)-diagnosed "DSM-IV Bipolar Disorder, Current Episode Manic" provided informed consent and participated in this study. Reports of hBPM differences between manic BD patients and healthy comparison participants can be found in our previously published work $[14,17,18]$; the participants in the current study were a subsample for which we had COMT genotypes available. Participants were between the ages of 18 and 55 years (mean age $=35.2$, standard deviation $=13.0$ ). Patients were excluded if they had abused or been dependent on alcohol or substances within the past month, had a positive result on a urine toxicology screen, had a neurological condition, or had a medical condition that impaired motor functioning. All patients were prescribed psychotropic medication during the time of testing, typically a combination of mood-stabilizing and atypical antipsychotic medications. The most common antipsychotic medication prescribed was risperidone and the most common mood stabilizers prescribed were lithium and valproate. Patients were administered the Young Mania Rating Scale (YMRS) to quantify manic symptoms. Patients' blood samples were analyzed for the COMT Val$158 \mathrm{Met}$ polymorphism per established methods [3]. Of the $26 \mathrm{pa}-$ tients, 6 were genotyped as Met/Met, 10 as Val/Val, and 10 as Val/ Met heterozygotes.

\section{Procedures}

Patients were tested in the hBPM, which has been described in detail elsewhere $[14,17]$. Briefly, the hBPM is a $2.7 \times 4.3 \mathrm{~m}$ room furnished with a desk, 2 bookcases, a standard filing cabinet, a short filing cabinet, 2 corkboards mounted on the walls, and vertical blinds covering the window. No chairs were placed in the room to encourage exploration. Eleven engaging toys (chosen using the 
criteria that they are safe, colorful, tactile, can be manipulated, and are likely to invite human exploration) were placed around the room. Patients were directed into the hBPM and asked to wait, but were not given other instructions or told how long their wait would be (15 min). Their activity was monitored by a digital video camera embedded in a ceiling vent. Video footage was scored by hand using a standardized manual for a second-by-second assessment of time spent with objects and interactions with more than 1 object at a time (MOIs). Raters were blind to diagnosis and to COMT genotype. We previously established interrater reliability coefficients of $0.91-0.96$ for the manualized scoring of hBPM videos. Additionally, as per our established methods, the hBPM was divided into a grid of 64 sectors. The number of entries into sectors proximal to novel objects, and time spent in these object-proximal sectors in seconds, were quantified using the $\mathrm{x}-\mathrm{y}$ coordinates of the subject's location.

\section{Statistical Analysis}

The three COMT genotype groups were compared on hBPM measures using one-way analyses of variance (ANOVA) with planned comparisons using $t$ tests. Effect sizes were calculated using Cohen's $d$. Additionally, the "dose" of the Met allele $(0=\mathrm{Val} /$ Val, $1=\mathrm{Val} /$ Met, 2 = Met/Met) was correlated with the hBPM measures using Pearson $r$ correlation coefficients; this dummycoding technique for the COMT genotype has been described previously [19]. Post hoc analyses were conducted using analysis of covariance (ANCOVA), Kruskal-Wallis tests, and Spearman rank correlations. Data were analyzed using SPSS 20 (Chicago, IL, USA).

\section{Results}

There were no significant differences in age or in the distribution of race, ethnicity, gender, YMRS scores, or chlorpromazine equivalents among the three genotype groups $(p>0.1)$ (Table 1), but Met/Met subjects did have lower mean YMRS scores and chlorpromazine equivalents than the other groups. A significant main effect of genotype was found for MOIs $(F(2,25)=4.4, p=0.03)$ with Met homozygote patients showing more MOIs $(d=$ $1.3, p=0.007)$ than Val homozygote patients. There was also a main effect of genotype on total time spent exploring objects $(F(2,25)=3.7, p=0.04)$, with Met homozygote patients showing more time spent with objects than either Val homozygote patients $(d=1.1, p=0.03)$ or heterozygote patients $(d=1.2, p=0.02)$. The dose of Met allele was significantly positively correlated with MOIs $(r=$ $0.52, p=0.007)$, total time exploring objects $(r=0.39, p=$

Fig. 1. The human Behavioral Pattern Monitor (hBPM) (a) and contour maps of entries into object-proximal sectors of the hBPM for Met/Met $(n=6)(\mathbf{b}), \mathrm{Val} / \mathrm{Met}(n=10)(\mathbf{c})$, and $\mathrm{Val} / \mathrm{Val}(n=10)$ (d) manic bipolar disorder (BD) patients.

COMT Val158Met Polymorphism and Exploratory Behavior in Bipolar Mania
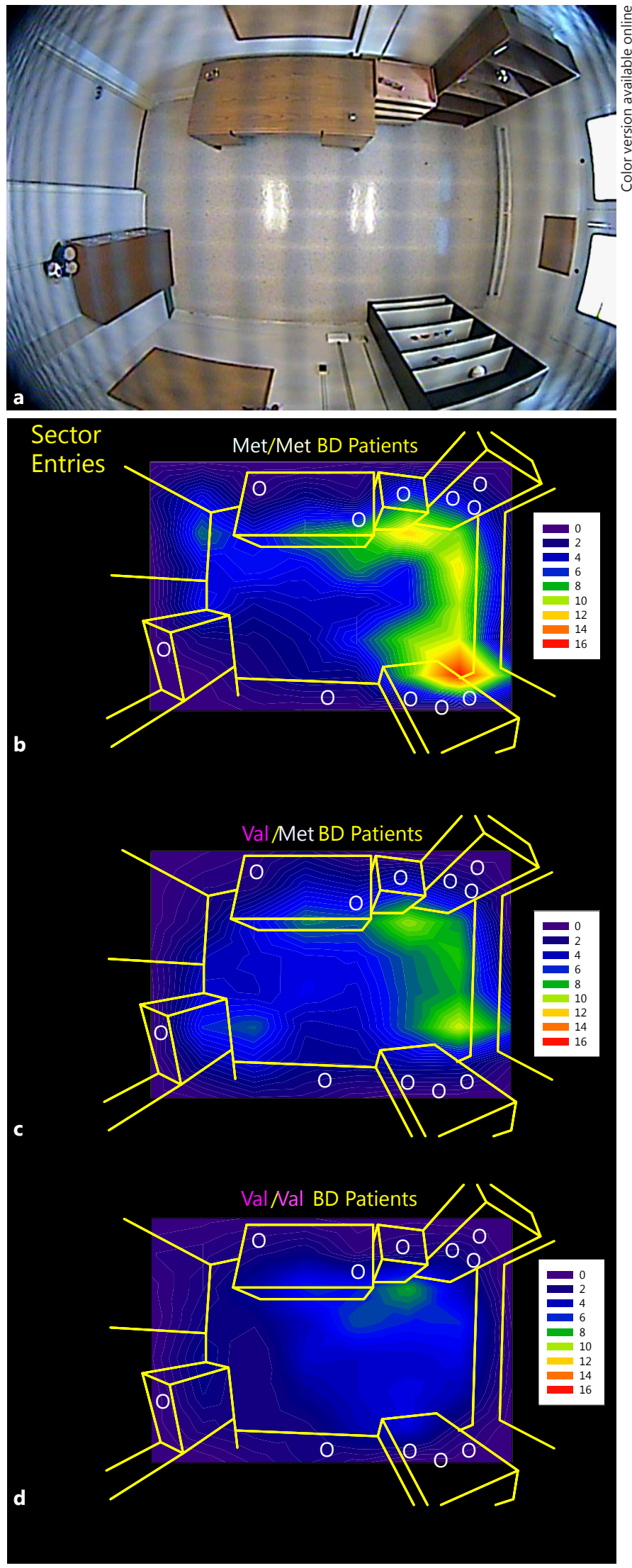

Mol Neuropsychiatry 2017;3:151-156 
Table 1. Demographic and symptom information for $\mathrm{Val} / \mathrm{Val}(n=10), \mathrm{Val} / \mathrm{Met}$ $(n=10)$, and Met/Met $(n=6)$ manic bipolar disorder patients

\begin{tabular}{lccc}
\hline & Val/Val & Val/Met & Met/Met \\
\hline Age, years & $36.8(12.0)$ & $33.8(14.3)$ & $34.7(14.6)$ \\
Education, years & $13.2(2.2)$ & $14.2(2.3)$ & $13.2(1.6)$ \\
Gender, $n$ & & & \\
$\quad$ Male & 6 & 3 & 3 \\
$\quad$ Female & 4 & 9 & 6 \\
Race, $n$ & & 0 & 0 \\
$\quad$ Caucasian & 7 & 1 & 0 \\
$\quad$ African American & 1 & 0 & 0 \\
$\quad$ Asian & 0 & 3 & 4 \\
$\quad$ More than 1 race & 2 & 7 & $23.3(8.9)$ \\
Ethnicity, $n$ & & & \\
$\quad$ Hispanic & 2 & $26.0(8.6)$ & $182.0(150.4)$ \\
$\quad$ Non-Hispanic & 8 & $249.9(352.3)$ & \\
YMRS scores & $31.4(6.0)$ & & \\
CPZ equivalents, mg & $316.6(365.2)$ & & \\
\hline
\end{tabular}

All data are means (standard deviations) unless otherwise noted. No group differences reached statistical significance. YMRS, Young Mania Rating Scale; CPZ, chlorpromazine.
$0.05)$, and time in object-proximal sectors $(r=0.41, p=$ 0.04) (Fig. 1).

Despite the lack of significant differences in race/ethnicity distribution among the COMT genotypes in this small sample, we noted that the Met homozygote subjects were all Caucasian (Table 1). Given this finding and the well-known ethnic differences in COMT allele frequency [20], we conducted the same analyses with just Caucasian subjects $(n=22)$. The direction of the findings remained the same, with the main effect of MOIs achieving statistical significance $(F(2,21)=4.1, p=0.03)$ and the main effect for time spent with objects approaching significance $(F(2,21)=3.3, p=0.06)$.

Because of the YMRS score and chlorpromazine equivalent differences between genotype groups, albeit not statistically significant, these variables were included as covariates in post hoc ANCOVAs. There remained a statistically significant effect of genotype on MOIs ( $p=$ $0.02-0.03$ ) and the effect of genotype on time spent with objects approached significance $(p=0.06-0.16)$. Additionally, because of the small sample size, post hoc nonparametric Kruskal-Wallis analyses were conducted; significant main effects of COMT genotype were observed for MOIs $\left(\chi^{2}=6.19, p=0.04\right)$ and time spent with objects $\left(\chi^{2}=6.70, p=0.04\right)$. Finally, Spearman rank correlations indicated a significant positive relationship between the dose of the Met allele and MOIs (rho $=0.49, p=0.01$ ), time spent with objects ( $r h o=0.40, p=0.04)$, and time in object-proximal sectors $(\mathrm{rho}=0.42, \mathrm{p}=0.04)$.

\section{Discussion}

In a small group of manic BD patients, we observed preliminary evidence that the Met allele of the COMT Val158Met polymorphism may relate to overactivity as measured by novel object exploration in the hBPM. Manic BD patients homozygous for the Met allele exhibited more interaction with multiple objects at the same time as well as increased time spent with objects. Most of these genotype differences remained significant when accounting for mania symptom scores and antipsychotic medication doses. Beyond observer ratings, video evidence also confirmed that time spent in regions of the hBPM that were proximal to objects were strongly related to the Met allele, as illustrated in the contour maps in Figure 1. These findings are consistent with the premise that the Met allele confers liability for increased motor arousal and positively valenced behaviors. Such behaviors are likely due to tonic increases in catecholamine levels in the frontal cortex which theoretically result in "difficulty in the smooth and effective modulation in response to novel or changing environmental exigencies" [10, p. 1,953]. Furthermore, the "dose" of the Met allele seems to be related in a linear fashion with this phenotype such that heterozygotic individuals demonstrate activity levels intermediate to the two homozygote groups. Hence, frontal synaptic DA clearance mechanisms are likely as important to behavioral abnormalities in $\mathrm{BD}$ as are striatal (e.g., DAT) mechanisms. It is important to note that the COMT Met 
allele is not universally associated with cognitive deficits; in fact, multiple studies in schizophrenia [21,22] as well as BD $[23,24]$ report that Val homozygote patients demonstrate the worst performance on cognitive domains such as working memory and executive function. These studies and our preliminary findings lend support to the $\mathrm{RDoC}$ taxonomy in that neurocognitive functions and the $\mathrm{RDoC}$-identified domains of arousal and positive valence do not appear to share entirely overlapping genetic underpinnings.

The role of the COMT Val158Met gene in phenotypes of mania and how it can be differentiated from schizophrenia has not been explored previously. An informal comparison of this cohort of manic BD patients to our previous sample of schizophrenia patients [14] suggests that, while Val homozygote patients exhibit the lowest levels of activity among $\mathrm{BD}$ mania patients, they remain more active than schizophrenia patients. Some authors have suggested that the presence of overactivity is as important as elevated and/or irritable mood for the diagnosis of mania and should be added as a core or stem criterion for manic states and be given priority over other symptoms [25], which occurred with the addition of "increased energy" as an essential criterion for a manic episode in DSM5. That YMRS scores did not differentiate among COMT genotypes (interestingly, it is Val homozygote patients, not Met, who have the highest YMRS scores), suggests a relationship between the Met allele and overactivity specific to behavioral domains and not the state of mania in general. Hence, these findings support the RDoC hypothesis that tying behavioral domains to circuits and genes may provide more targeted therapeutics in the future.

Despite large effect sizes, the current findings are preliminary and in need of replication in a larger sample; furthermore, we recognize that underpowered studies may overestimate effect magnitude [26]. The genotype groups did differ in manic symptom scores and antipsychotic medication equivalencies, with Val homozygote patients having higher YMRS scores and on average higher doses of antipsychotic medication. Although these differences did not reach statistical significance and likely do not entirely explain the genotype differences in hBPM activity, they do suggest that these groups were not equally ill. This and other potential confounds cannot be entirely ruled out in this small candidate gene study.

Additionally, our results do not address whether the gene-behavior associations seen here are specific to mania or whether they exist in other phases of $\mathrm{BD}$, or in other conditions with putative catecholamine dysregulation such as $\mathrm{ADHD}$, schizophrenia, substance use conditions, and, importantly, in healthy individuals. If, for example, we did not observe the same effect of COMT on motor activity in healthy subjects, we might postulate an additive effect of genetic predisposition and a psychiatric condition which putatively confers catecholamine overactivity. Such an effect is arguably consistent with the proposed inverted U-shaped function of COMT (see [27] for a review), where the two sources of catecholamine overactivity increase the signal-to-noise ratio of catecholamine function in the prefrontal cortex, with adverse consequences. As an illustration of this effect, Mattay et al. [28] demonstrated that healthy Met homozygote subjects given amphetamine performed poorly on an executive functioning task, while amphetamine improved the performance of Val homozygote subjects (but see [29] for a contrasting finding). It would be interesting to assess whether the COMT Met allele is associated with changes in the exploratory behavior phenotype we have observed in the hBPM in subjects with a history of methamphetamine dependence [30]. Such findings would provide even greater support for the RDoC mission of uncovering a phenotype-biomarker relationship that cuts across psychiatric conditions.

Genetic manipulations have been used extensively to quantify alterations in rodent activity and exploration over the past two decades. The current findings represent evidence that we can describe the influence of genes on behavior using the hBPM, a paradigm that enables human and rodent cross-species comparisons. The use of such translational paradigms allows for the development of novel, perhaps genotype-based, therapeutics for $\mathrm{BD}$ and other conditions characterized by hallmark and measurable phenotypes.

\section{Acknowledgments}

The authors acknowledge the contributions of Virginia Masten, Christin Seed, and Tatyana Shekhtman to this work.

\section{Disclosure Statement}

The authors report no financial disclosures. Other disclosures: Dr. Kelsoe serves on the Editorial Board of Molecular Neuropsychiatry.

\section{Funding Sources}

This work was supported by a grant from the National Institute of Mental Health (NIMH) (R01-MH071916) and a NARSAD Young Investigator Award (A.M.). 


\section{References}

1 Insel TR: The NIMH Research Domain Criteria (RDoC) Project: precision medicine for psychiatry. Am J Psychiatry 2014;171:395397.

2 Lyon M: Animal models for the symptoms of mania; in Boulton AA, Baker GB, MartinIverson MT (eds): Animal Models in Psychiatry. Clifton, Humana Press, 1991, pp 197-244.

3 Greenwood TA, Schork NJ, Eskin E, Kelsoe JR: Identification of additional variants within the human dopamine transporter gene provides further evidence for an association with bipolar disorder in two independent samples. Mol Psychiatry 2006;11:115, 125133.

4 Rivett AJ, Eddy BJ, Roth JA: Contribution of sulfate conjugation, deamination, and $\mathrm{O}$ methylation to metabolism of dopamine and norepinephrine in human brain. J Neurochem 1982;39:1009-1016.

5 Lachman HM, Papolos DF, Saito T, Yu YM, Szumlanski CL, Weinshilboum RM: Human catechol-O-methyltransferase pharmacogenetics: description of a functional polymorphism and its potential application to neuropsychiatric disorders. Pharmacogenetics 1996;6:243-250.

6 Phillips ML, Swartz HA: A critical appraisal of neuroimaging studies of bipolar disorder: toward a new conceptualization of underlying neural circuitry and a road map for future research. Am J Psychiatry 2014;171:829-843.

7 Quednow BB, Wagner M, Mossner R, Maier W, Kuhn KU: Sensorimotor gating of schizophrenia patients depends on catechol Omethyltransferase Val158Met polymorphism. Schizophr Bull 2010;36:341-346.

8 Kotler M, Barak P, Cohen H, Averbuch IE, Grinshpoon A, Gritsenko I, Nemanov L, Ebstein RP: Homicidal behavior in schizophrenia associated with a genetic polymorphism determining low catechol O-methyltransferase (COMT) activity. Am J Med Genet 1999 88:628-633.

9 Nolan KA, Volavka J, Czobor P, Cseh A, Lachman H, Saito T, Tiihonen J, Putkonen A, Hallikainen T, Kotilainen I, Rasanen P, Isohanni M, Jarvelin MR, Karvonen MK: Suicidal behavior in patients with schizophrenia is related to COMT polymorphism. Psychiatr Genet 2000;10:117-124.

10 Bilder RM, Volavka J, Lachman HM, Grace AA: The catechol-O-methyltransferase polymorphism: relations to the tonic-phasic dopamine hypothesis and neuropsychiatric phenotypes. Neuropsychopharmacology 2004 29:1943-1961.
11 Swann AC, Lijffijt M, Lane SD, Steinberg JL, Moeller FG: Severity of bipolar disorder is associated with impairment of response inhibition. J Affect Disord 2009;116:30-36.

12 Geyer MA, Russo PV, Masten VL: Multivariate assessment of locomotor behavior: pharmacological and behavioral analyses. Pharmacol Biochem Behav 1986;25:277-288.

13 Perry W, Minassian A, Henry B, Kincaid M, Young JW, Geyer MA: Quantifying over-activity in bipolar and schizophrenia patients in a human open field paradigm. Psychiatry Res 2010;178:84-91.

14 Perry W, Minassian A, Paulus MP, Young JW, Kincaid MJ, Ferguson EJ, Henry BL, Zhuang X, Masten VL, Sharp RF, Geyer MA: A reverse-translational study of dysfunctional exploration in psychiatric disorders: from mice to men. Arch Gen Psychiatry 2009;66: 1072-1080.

15 Morris SE, Cuthbert BN: Research Domain Criteria: cognitive systems, neural circuits, and dimensions of behavior. Dialogues Clin Neurosci 2012;14:29-37.

16 Young JW, Minassian A, Paulus MP, Geyer MA, Perry W: A reverse-translational approach to bipolar disorder: rodent and human studies in the Behavioral Pattern Monitor. Neurosci Biobehav Rev 2007;31:882-896.

17 Minassian A, Henry BL, Geyer MA, Paulus MP, Young JW, Perry W: The quantitative assessment of motor activity in mania and schizophrenia. J Affect Disord 2010;120:200206.

18 Perry W, McIlwain M, Kloezeman K, Henry BL, Minassian A: Diagnosis and characterization of mania: quantifying increased energy and activity in the human behavioral pattern monitor. Psychiatry Res 2016;240:278-283.

19 Bilder RM, Volavka J, Czobor P, Malhotra AK, Kennedy JL, Ni X, Goldman RS, Hoptman MJ, Sheitman B, Lindenmayer JP, Citrome L, McEvoy JP, Kunz M, Chakos M, Cooper TB, Lieberman JA: Neurocognitive correlates of the COMT Val(158)Met polymorphism in chronic schizophrenia. Biol Psychiatry 2002;52:701-707.

20 McLeod HL, Fang L, Luo X, Scott EP, Evans WE: Ethnic differences in erythrocyte catechol-O-methyltransferase activity in black and white Americans. J Pharmacol Exp Ther 1994;270:26-29.

21 Egan MF, Goldberg TE, Kolachana BS, Callicott JH, Mazzanti CM, Straub RE, Goldman D, Weinberger DR: Effect of COMT Val108/158 Met genotype on frontal lobe function and risk for schizophrenia. Proc Natl Acad Sci USA 2001;98:6917-6922.
22 Goldberg TE, Egan MF, Gscheidle T, Coppola R, Weickert T, Kolachana BS, Goldman D, Weinberger DR: Executive subprocesses in working memory: relationship to catechol-Omethyltransferase Val158Met genotype and schizophrenia. Arch Gen Psychiatry 2003;60: 889-896.

23 Dickerson FB, Boronow JJ, Stallings C, Origoni AE, Cole S, Leister F, Krivogorsky B, Yolken RH: The catechol O-methyltransferase Val158Met polymorphism and herpes simplex virus type 1 infection are risk factors for cognitive impairment in bipolar disorder: additive gene-environmental effects in a complex human psychiatric disorder. Bipolar Disord 2006;8:124-132.

24 Miskowiak KW, Kjaerstad HL, Stottrup MM, Svendsen AM, Demant KM, Hoeffding LK, Werge TM, Burdick KE, Domschke K, Carvalho AF, Vieta E, Vinberg M, Kessing LV, Siebner HR, Macoveanu J: The catechol-Omethyltransferase (COMT) Val158Met genotype modulates working memory-related dorsolateral prefrontal response and performance in bipolar disorder. Bipolar Disord 2017;19:214-224.

25 Benazzi F: Is overactivity the core feature of hypomania in bipolar II disorder? Psychopathology 2007;40:54-60.

26 Button KS, Ioannidis JP, Mokrysz C, Nosek BA, Flint J, Robinson ES, Munafo MR: Power failure: why small sample size undermines the reliability of neuroscience. Nat Rev Neurosci 2013;14:365-376.

27 Meyer-Lindenberg A, Weinberger DR: Intermediate phenotypes and genetic mechanisms of psychiatric disorders. Nat Rev Neurosci 2006;7:818-827.

28 Mattay VS, Goldberg TE, Fera F, Hariri AR, Tessitore A, Egan MF, Kolachana B, Callicott JH, Weinberger DR: Catechol O-methyltransferase val158-met genotype and individual variation in the brain response to amphetamine. Proc Natl Acad Sci USA 2003;100: 6186-6191.

29 Wardle MC, Hart AB, Palmer AA, de Wit H: Does COMT genotype influence the effects of d-amphetamine on executive functioning? Genes Brain Behav 2013;12:13-20.

30 Henry BL, Minassian A, van Rhenen M, Young JW, Geyer MA, Perry W: Effect of methamphetamine dependence on inhibitory deficits in a novel human open-field paradigm. Psychopharmacology (Berl) 2011;215: 697-707. 